\title{
Heart rate variability and long chain n-3 PUFA dietary intake and status: a comparison between matched vegans and omnivores
}

\author{
A.M. Pinto, H.K. Al Khatib, T.A.B. Sanders and W.L. Hall \\ Diabetes \& Nutritional Sciences Division, Faculty of Life Sciences \& Medicine, King's College London, Franklin \\ Wilkins Building, 150 Stamford Street, London, SE1 9NH
}

Heart rate variability (HRV) refers to a range of parameters that reflect variability in interbeat intervals (IBI), and is an indirect measure of cardiac autonomic function. Low HRV is a powerful predictor of sudden cardiac death ${ }^{(1)}$. HRV has been positively associated with long chain n-3 PUFA tissue content, mainly docosahexaenoic acid (DHA), which is a component of myocardial membranes ${ }^{(2)}$. We hypothesise that lower n-3 PUFA status in vegan subjects, whose diets are devoid of eicosapentaenoic acid (EPA) and DHA, might be associated with lower HRV compared to omnivores. This cross-sectional study aimed to compare HRV between nonsmoking vegans and sex/age/BMI-matched omnivores (40-70 y).

Measurements of $24 \mathrm{~h} \mathrm{HRV}$ were made using Actiheart monitors (CamNtech Ltd, Cambridge, UK). The Actiheart device provides IBI data of similar quality to a Holter monitor but also records movement using an accelerometer. The following $24 \mathrm{~h}$ HRV parameters (frequency and time domain) are reported adjusted for age, sex, BMI and physical activity: SDNN is an estimate of overall variability; SDANN and very low frequency (VLF) power indicate longer-phase variability. Plasma and erythrocyte fatty acid composition were analysed by GC-MS and a food frequency questionnaire was used to estimate dietary fatty acid intake.

\begin{tabular}{|c|c|c|c|c|c|}
\hline & Omnivore $(n=24)$ & Vegan $(n=23)$ & Mean $\Delta$ & $95 \% \mathrm{CI}$ & $P$ value ${ }^{\dagger}$ \\
\hline \multicolumn{6}{|c|}{ Erythrocyte fatty acid composition (weight \%) } \\
\hline $\mathbf{L A}$ & $11 \cdot 7(11 \cdot 0,12 \cdot 3)$ & $13 \cdot 3(12 \cdot 5,14 \cdot 1)$ & 1.64 & $(0.64,2.64)$ & 0.002 \\
\hline $\mathbf{A} \mathbf{L} \mathbf{A}^{\mathbf{b}}$ & $0 \cdot 34(0 \cdot 26,0 \cdot 45)$ & $0.32(0.27,0.38)$ & 0.07 & $(-0 \cdot 20,0 \cdot 08)$ & $0 \cdot 610$ \\
\hline EPA & $1.26(1.07,1.45)$ & $0.67(0.52,0.81)$ & -0.59 & $(-0.83,-0.36)$ & $<0.001$ \\
\hline DHA $^{b}$ & $4.19(3.63,4.83)$ & $2 \cdot 07(1.85,2.32)$ & $-2 \cdot 27$ & $(-2.86,-1.67)$ & $<0.001$ \\
\hline \multicolumn{6}{|c|}{ Dietary fatty acid intake $(\% \mathrm{E})$} \\
\hline LA & $3 \cdot 2(2 \cdot 8,3 \cdot 6)$ & $6 \cdot 4(5 \cdot 0,7 \cdot 8)$ & $-\mathbf{3} \cdot \mathbf{2}$ & $(-4 \cdot 6,-1 \cdot 8)$ & $<0.001^{\mathrm{a}}$ \\
\hline ALA & $0.29(0.25,0.32)$ & $0.92(0.25,1.59)$ & -0.64 & $(-1.27,0.00)$ & $0.017^{\mathrm{a}}$ \\
\hline EPA & $0.07(0.06,0.09)$ & $0.00(0.00,0.00)$ & 0.07 & $(0.06,0.09)$ & $<0.001^{\mathrm{a}}$ \\
\hline DHA & $0.30(0.20,0.41)$ & $0.00(0.00,0.00)$ & $\mathbf{0} \cdot \mathbf{3 0}$ & $(0 \cdot 19,0 \cdot 41)$ & $<0.001^{\mathrm{a}}$ \\
\hline \multicolumn{6}{|c|}{ HRV measurements (24 h) } \\
\hline IBI (ms) & $834(791,877)$ & $795(750,840)$ & -38 & $(-103,26)$ & 0.237 \\
\hline SDNN (ms) & $144(129,158)$ & $167(152,182)$ & $23 \cdot 6$ & $(1 \cdot 9,45 \cdot 3)$ & $\mathbf{0 . 0 3 4}$ \\
\hline SDANN (ms) & $125(111,134)$ & $154(140,169)$ & 29.4 & $(8 \cdot 4,50 \cdot 4)$ & 0.007 \\
\hline $\operatorname{VLF}\left(\mathrm{ms}^{2}\right)^{\mathbf{b}}$ & $12308(10142,14932)$ & $17588(14360,21538)$ & 6027 & $(1102,10953)$ & 0.017 \\
\hline
\end{tabular}

Results expressed as mean $(95 \% \mathrm{CI})$. ALA, $\alpha$-linolenic acid; EPA, eicosapentaenoic acid; LA, linoleic acid ${ }^{\mathrm{a}}$ Using Mann-Whitney $\mathrm{U}$ test. ${ }^{\mathrm{b}} \mathrm{Geometric}$ mean. ${ }^{\dagger}$ Using independent samples $t$-test.

SDNN, SDANN and VLF were significantly higher in vegans. There were no differences in HRV parameters reflecting shorterphase variability. The proportions of both EPA and DHA in erythrocyte membranes and plasma were significantly lower in vegans whereas LA was significantly higher. Although there was no difference in ALA in erythrocytes, the proportion of total plasma fatty acids as ALA was significantly higher in vegans (mean difference $0.23 \% \mathrm{wt}$; $95 \%$ CI $0 \cdot 06,0 \cdot 41$ ).

These data disprove the hypothesis that vegans would have reduced HRV linked to their lower long chain n-3 PUFA status. In fact, vegans had increased parameters of overall and longer-term HRV compared to omnivores. This suggests that other factors associated with a vegan diet and overall lifestyle might offset the cardiovascular autonomic consequences of low EPA + DHA tissue status.

1. Malik M, Bigger JT, Camm AJ et al. (1996) Task Force of the European Society of Cardiology, and the North American Society of Pacing, and Electrophysiology. Circulation 93, 1043-1065.

2. Christensen JH, Svensson M, Strandhave C et al. (2010) N-3 Fatty acids and cardiac autonomic function in humans. Cell Mol Biol 56, 131-139. 